\title{
Comparison of Wavelet Packet and Wavelet in Solving Arbitrary Array of Parallel Wires Integral Equations in Electromagnetics
}

\author{
Mohamed Bayjja 1, Gamil Alsharahi 1, Mohamed Aghoutane 1, Naima Amar Touhami 1 \\ ${ }^{1}$ Department Physics, Faculty of Science, University Abdelmalek Essaâdi, Tetouan, Morocco.
}

Corresponding author: Mohamed Bayjja (e-mail: m.bayjja@gmail.com).

\begin{abstract}
In this paper, wavelets transformation (WT) and wavelet packet transformation (WPT) are used in solving, by the method of moments, a semicircular array of parallel wires electric field integral equation. First, the integral equation is solved by applying the direct method of moments via pointmatching procedure, results in a linear system with a dense matrix. Therefore, wavelet transformation and wavelet packet transformation are used to sparsify the impedance matrix, using two categories of wavelets functions, Biorthogonal (bior2.2) and Orthogonal (db4) wavelets. The far-field scattering patterns and the comparison between wavelets transformation and wavelet packet transformation in term number of zeros in impedance matrix and CPU Time reduction are presented. Numerical results are presented to identify which technique is best suited to solve such scattering electromagnetic problems and compared with published results.
\end{abstract}

INDEX TERMS: Arbitrary Array of Parallel Wires, Moment Method, Integral equation, Sparse Matrix, Wavelets, Wavelet Packet.

\section{INTRODUCTION}

$\mathbf{T}$ he art of computation of electromagnetic (EM) problems has grown exponentially for three decades due to the availability of powerful computer resources. Solving electromagnetic problems requires the application of Maxwell's Equations with the appropriate formulation and boundary conditions. Many powerful numerical analysis techniques have been developed in this area in the last 50 years $[1,4-5]$.

The use of method of moments (MoM) in EM has become popular since the work of Richmond in 1965 and Harrington [7] in 1967. The method has been successfully applied to a wide variety of EM problems of practical interest such as analysis of electromagnetic wave scattering, radiating, and guiding problems, to mention a few. In conventional MoM, the unknown function is expanded in terms of known basis functions with unknown coefficients. Unfortunately, the conventional bases, trigonometric, Legendre, Bessel, Hermite, and Chebyshev, when applied directly to the integral equations, generally lead to a dense matrix. As a result, the inversion and the final solution of such a system of linear equations are very time consuming [7].
There has been an explosive growth of research on wavelets, particularly in the last decade, resulting in a plethora of books, journals, and research papers dealing with the topic [8]. Recently, to fix the poor frequency localization of high frequency wavelet bases, Coifman, Meyer, and Wickerhauser [1-6] have generalized the construction of many other orthonormal bases of timefrequency atoms, corresponding to different tilings of the time-frequency plane. Wavelet packet base is one important example constructed, with time-frequency atoms that split the frequency and the time axis, respectively, in intervals of varying sizes. Wavelet packet bases are designed by dividing the frequency axis in intervals of varying sizes. These bases are particularly well adapted to decomposing signals that have different behavior in different frequency intervals [1-12]. The wavelets are a powerful technique for solving integral equations, resulting in sparse impedance matrices [1]. This is due to features of vanishing moments, orthogonality and multiresolution analysis in wavelets. The wavelet approach is used directly on the impedance matrix in order to make it sparse rather than on the operator equations in the form of a set of basis functions to approximate the unknown. The conversion of dense 
matrices into a sparse form requires $\mathrm{O}\left(\mathrm{N}^{2}\right)$ operations. The algorithm for solving the resulting sparse system requires only $\mathrm{O}(\mathrm{N} \log 2 \mathrm{~N})$ operations, as shown in [12].

This work is concerned to study of wavelets and wavelet packets for the Solution of Electromagnetic Integral Equations. The paper is divided into three main parts; first the problems of a plane wave by an arbitrary array of parallel wires and scattering are presented. The next section briefly presents the matrix formulation of wavelet transform and we discuss wavelet packets. The numerical examples suggesting sparsity of the transformed matrices and comparisons between wavelet and wavelet packets. The last section contains conclusions and suggestions for future research.

\section{PROBLEM FORMULATION}

\section{A. BASIC THEORY of SCATTERING by an ARBITRARY ARRAY of PARALLEL WIRES}

Consider the problem of computing the scattering of a TM polarized electromagnetic wave from a 2-D an Arbitrary Array of Parallel Wires with the boundary contour. This problem is of more general nature than the scattering wire and antenna wire [2-3]. The far-field scattering characteristics are obtained from the surface current excited by an incident wave. An arbitrary array of $\mathrm{N}$ parallel, circular wires of infinite length placed parallel to the z-axis are assumed to exist in unbounded free space is illustrated in Fig. 1 [2-3]. Let a harmonic TM wave be incident on the wires. Assuming a time factor $\mathrm{e}^{\mathrm{j} \omega \mathrm{t}}$, the incident wave in phasor form is given by

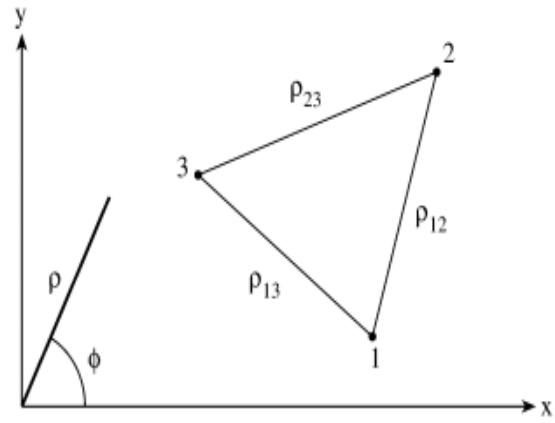

(a)

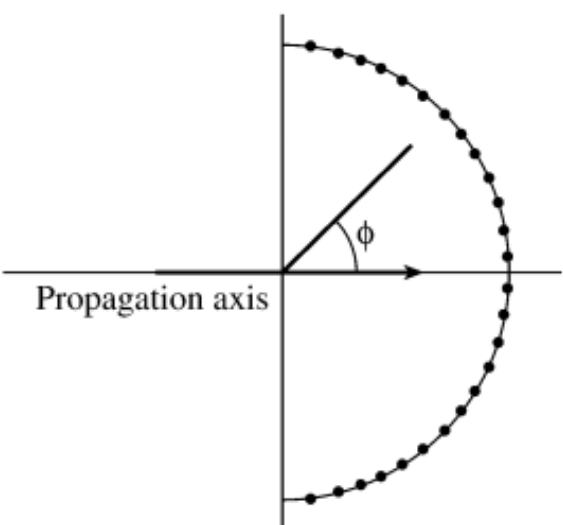

(b)

FIGURE 1. (a) An array of three wires parallel to the z-axis, (b) a semicircular array of 30 parallel wires.

Let a harmonic TM wave be incident on the wires. Assuming a time factor $\mathrm{e}^{\mathrm{j} \omega \mathrm{t}}$, the incident wave in phasor form is given by [2]

$$
H_{z}^{i}=0
$$

And

$$
E_{z}^{i}=E_{i}(x, y) e^{-i h z}
$$

The incident wave may be, for example, one or more plane waves or a continuous spectrum of plane waves traveling in different directions, If a plane wave has an axis of propagation which makes an angle $\varphi 0$ with the $\mathrm{z}$ axis, its field is given by

$$
\begin{gathered}
E_{i}(x, y)=E_{0} e^{-i k\left(x \sin \theta_{i} \cos \varphi_{i}+y \sin \theta_{i} \sin \varphi_{i}\right)} \\
h=k \cos \theta_{i} \\
k=\frac{2 \pi}{\lambda}
\end{gathered}
$$

The angle $\theta \mathrm{i}$ and $\varphi \mathrm{i}$, illustrated in Fig. 2, are the angular coordinates of the axis of propagation in the spherical coordinate system. The incident wave induces current on the surface of wire $n$.

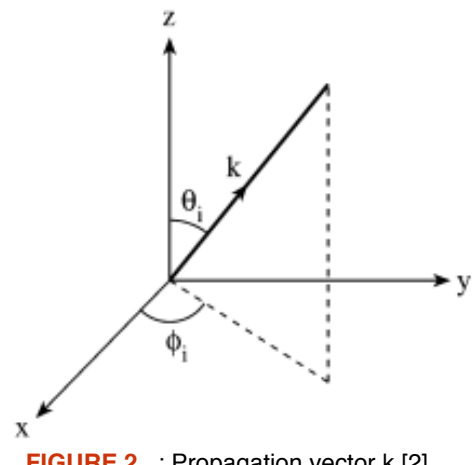


It can readily be shown that the field of a harmonic current In uniformly distributed on a circular cylinder of radius $a_{n}$ has a $\mathrm{z}$ component given by [2-3]

$$
E_{n}=-I_{n}^{\prime} H_{0}^{(2)}\left(g \rho_{n}\right) e^{-i h z} \quad \rho_{n}>a_{n}
$$

Where

$$
\begin{gathered}
-\mathrm{I}_{\mathrm{n}}^{\prime}=\frac{\omega \mu \mathrm{g}^{2}}{4 \mathrm{k}^{2}} \mathrm{I}_{\mathrm{n}} \mathrm{J}_{0}\left(\mathrm{ga}_{\mathrm{n}}\right) \\
\mathrm{g}^{2}+\mathrm{h}^{2}=\mathrm{k}^{2}
\end{gathered}
$$

$\mathrm{J}_{0}$ is Bessel function of order zero, and $\mathrm{H}_{0}$ is Hankel function of the second kind of order zero. By induction theorem, if $I n$ is regarded as the induced current, Eq. (7) may be considered as the scattered field, i.e.

$$
E_{z}^{S}=-\sum_{n=1}^{N} I_{n}^{\prime} H_{0}^{(2)}\left(g \rho_{n}\right) e^{-i h z}
$$

Where the summation is taken over all the $\mathrm{N}$ wires. On the surface of each wire (assumed perfectly conducting),

$$
E_{z}^{i}=-E_{z}^{S}, \quad \rho=\rho_{n}
$$

Substitution of Eqs. (9) and (7) into Eq. (10) leads to N,

$$
\sum_{n=1}^{N} I_{n}^{\prime} H_{0}^{(2)}\left(g \rho_{m n}\right)=E_{z}^{i}\left(x_{m}, y_{m}\right)
$$

Where

$$
\rho_{m n}= \begin{cases}\sqrt{\left(x_{m}-x_{n}\right)^{2}+\left(y_{m}-y_{n}\right)^{2}}, & m \neq n \\ a_{m} & , m=n\end{cases}
$$

And $\mathrm{a}_{\mathrm{m}}$ is the radius of the $m t h$ wire. In matrix fro, Eq.(11) can be written as

$$
[A][I]=[B]
$$

Or

$$
[I]=[A]^{-1}[B]
$$

Where

$$
\begin{gathered}
I_{n}=I_{n}^{\prime} \\
A_{m n}=H_{0}^{(2)}\left(g \rho_{m n}\right) \\
B_{m}=E_{0} e^{-i k\left(x_{m} \sin \theta_{i} \cos \varphi_{i}+x_{m} \sin \theta_{i} \sin \varphi_{i}\right)}
\end{gathered}
$$

Once $I_{n}^{\prime}$ is calculated from Eq. 14 , the scattered field can be obtained as

$$
E_{z}^{S}=-\sum_{n=1}^{N} I_{n}^{\prime} H_{0}^{(2)}\left(g \rho_{n}\right) e^{-i h z}
$$

Finally, we may calculate the "distant scattering pattern," defined as

$$
E(\varphi)=\sum_{n=1}^{N} I_{n}^{\prime} e^{-i g(x n \cos \varphi+y n \sin \varphi)}
$$

The following example, taken from Richmond's work [3], will be used to illustrate the techniques discussed in the latter half of this section.

\section{B. The WAVELET TRANSFORM}

The idea behind applying wavelets to the Moment Method is to decrease the size of the discretization matrix of the integral equation. This approach is made by applying wavelets to the matrix obtained after discretization by the fast wavelet transform (FWT). Orthogonal wavelets have several properties such as orthonormal bases of $\mathrm{L}^{2}(\mathrm{R})$, trade-off between orthogonality and continuity, order of regularity, and cancellation property, that are fascinating for electromagnetic field computations. Furthermore, orthogonal wavelets have localization properties in both the spatial and spectral domains [1,7-8].

In this section, the impedance matrix (IM) obtained by $\mathrm{MoM}$ is reduced to sparse one by a wavelet matrix transform and, hence, the computation cost is relatively lowered. By using the MoM, we obtain the matrix equation as Eq.13, where [Z] is a dense impedance matrix. Introducing a wavelet matrix [W], the matrix equation is then transformed as [8]:

$$
[\mathrm{Z}]^{\prime}[\mathrm{I}]^{\prime}=[\mathrm{V}]^{\prime}
$$

$$
\mathrm{Z}^{\prime}=\mathrm{WZW}^{\mathrm{T}}, \mathrm{I}^{\prime}=\left(\mathrm{W}^{\mathrm{T}}\right)^{\prime} \mathrm{I} \text {, and } \mathrm{V}^{\prime}=\mathrm{WV}
$$

Here $T$ stands for the transpose of a matrix. One [I]' is solved; the desired solution is obtained as:

$$
[\mathrm{I}]=[\mathrm{W}]^{\mathrm{T}}[\mathrm{I}]^{\prime}
$$

Z' has numerous very small elements which can be neglected (threshold) without largely affecting the solution. Practical considerations require that matrices must satisfy the following design criteria [8].

- The matrix must be (effectively) sparse.

- Matrices must be sparse, so that matrix-matrix multiplications cost only.

- The condition number of is not much larger than that of.

The last criterium is dictated by the fact that iterative methods converge more slowly for systems with larger condition numbers.

The continuous operator and discrete operator are related by

$$
\mathrm{Z}=\lim _{j \rightarrow \infty} P_{j} Z P_{j}=\lim _{j \rightarrow \infty} V_{j}
$$


where $\mathrm{Z}^{\mathrm{j}}$ is the approximation of $\mathrm{Z}$ projected on $\mathrm{Vj}$ and tested in $\mathrm{Vj}$. The nonstandard form method toward the sparsification of an existing impedance matrix is:

Since:

$$
\begin{array}{r}
V_{j+1}=V_{j} \oplus W_{j} \\
Z^{j+1}=\left[\begin{array}{cc}
A^{j j} & B^{j j} \\
C^{j j} & Z_{k k^{\prime}}^{j}
\end{array}\right]
\end{array}
$$

Where,

$$
\begin{aligned}
& \mathrm{A}_{\mathrm{kk}{ }^{\prime}}^{\mathrm{jj}}=\left\langle\Psi_{\mathrm{j}, \mathrm{k}}, \mathrm{Z}\left(\Psi_{\mathrm{j}, \mathrm{k}^{\prime}}\right)\right\rangle \\
& \mathrm{B}_{\mathrm{kk}{ }^{\prime}}^{\mathrm{jj}}=\left\langle\psi_{\mathrm{j}, \mathrm{k}}, \mathrm{Z}\left(\varphi_{\mathrm{j}, \mathrm{k}^{\prime}}\right)\right\rangle \\
& \mathrm{C}_{\mathrm{kk}{ }^{\mathrm{j}}}^{\mathrm{jj}}=\left\langle\varphi_{\mathrm{j}, \mathrm{k}}, \mathrm{Z}\left(\Psi_{\mathrm{j}, \mathrm{k}^{\prime}}\right)\right\rangle \\
& \mathrm{Z}_{\mathrm{kk} \mathrm{k}^{\prime}}^{\mathrm{j}}=\left\langle\varphi_{\mathrm{j}, \mathrm{k}}, \mathrm{Z}\left(\varphi_{\mathrm{j}, \mathrm{k}^{\prime}}\right)\right\rangle
\end{aligned}
$$

Matrix $A_{\mathrm{kk} \prime}^{\mathrm{jj}}$ is very sparse because both of the expansion and testing functions are wavelets. Matrices $\mathrm{B}_{\mathrm{kk}}^{\mathrm{jj}}$, and $\mathrm{C}_{\mathrm{kk} \text { }}^{\mathrm{jj}}$ are composed of a mix of scalet and wavelet. Matrix $\mathrm{Z}_{\mathrm{kk} \mathrm{k}^{\prime}}^{\mathrm{j}}$ is dense because both of the expansion and testing functions are scalets. These submatrices represent the interaction between the sources and fields in different subspaces [8]. Submatrix A, B, C, and $\mathrm{Z}$ of the matrix $\mathrm{Z}$ can be evaluated as follows.

$$
\begin{aligned}
& \mathrm{A}_{\mathrm{kk}{ }^{\prime}}^{\mathrm{jj}}=\sum_{\mathrm{m}} \sum_{\mathrm{n}} \mathrm{g}_{\mathrm{n}-2 \mathrm{k}} \mathrm{g}_{\mathrm{m}-2 \mathrm{k}^{\prime}}\left\langle\varphi_{\mathrm{j}+1, \mathrm{n}}, \mathrm{Z}\left(\varphi_{\mathrm{j}+1, \mathrm{~m}}\right)\right\rangle \\
& \mathrm{B}_{\mathrm{kk},}^{\mathrm{jj}}=\sum_{\mathrm{n}} \sum_{\mathrm{m}} \mathrm{g}_{\mathrm{n}-2 \mathrm{k}} \mathrm{h}_{\mathrm{m}-2 \mathrm{k}} \mathrm{z}_{\mathrm{n}, \mathrm{m}}^{\mathrm{j}+1} \\
& \mathrm{C}_{\mathrm{kk} \prime}^{\mathrm{jj}}=\sum_{\mathrm{n}} \sum_{\mathrm{m}} \mathrm{h}_{\mathrm{n}-2 \mathrm{k}} \mathrm{g}_{\mathrm{m}-2 \mathrm{k}} \mathrm{z}_{\mathrm{n}, \mathrm{m}}^{\mathrm{j}+1} \\
& \mathrm{Z}_{\mathrm{kk} \mathrm{k}^{\prime}}^{\mathrm{j}}=\sum_{\mathrm{n}} \sum_{\mathrm{m}} \mathrm{h}_{\mathrm{n}-2 \mathrm{k}} \mathrm{h}_{\mathrm{m}-2 \mathrm{k},} \mathrm{z}_{\mathrm{n}, \mathrm{m}}^{\mathrm{j}+1}
\end{aligned}
$$

The development of detailed steps relies on the two equations

$$
\begin{aligned}
\varphi_{\mathrm{j}, \mathrm{k}} & =\sum_{\mathrm{n}} \mathrm{h}_{\mathrm{n}-2 \mathrm{k}} \varphi_{\mathrm{j}+1, \mathrm{n}} \\
\psi_{\mathrm{j}, \mathrm{k}} & =\sum_{\mathrm{n}} \mathrm{g}_{\mathrm{n}-2 \mathrm{k}} \varphi_{\mathrm{j}+1, \mathrm{n}}
\end{aligned}
$$

Where $\mathrm{h}$ and $\mathrm{g}$ are the lowpass and bandpass filter coefficients, respectively.

We may further improve the matrix sparsity in the nonstandard form as follows. Let us consider the lower-left quarter, $C j j$. Notice the fact that

$$
\begin{gathered}
\mathrm{C}^{\mathrm{l}, \mathrm{j}}=\left(\begin{array}{l}
\mathrm{A}^{\mathrm{l}-1, \mathrm{j}} \\
\mathrm{C}^{\mathrm{l}-1, \mathrm{j}}
\end{array}\right) \quad l=j, j-1, \ldots \ldots, 1 . \\
\mathrm{A}_{\mathrm{k}, \mathrm{k} \mathrm{k}^{\prime}}^{\mathrm{l-1, \textrm {j }}}=\left\langle\psi_{\mathrm{l}-1, \mathrm{k}}, \mathrm{Z}\left(\Psi_{\mathrm{j}, \mathrm{k}^{\prime}}\right)\right\rangle \\
=\sum_{n} \mathrm{~g}_{\mathrm{n}-2 \mathrm{k}} \mathrm{C}_{\mathrm{n}, \mathrm{k}^{\prime}}^{\mathrm{l,j}}
\end{gathered}
$$

With;

$$
\mathrm{C}_{\mathrm{k}, \mathrm{k} \prime}^{\mathrm{l}-1, \mathrm{j}}=\sum_{n} \mathrm{~h}_{\mathrm{n}-2 \mathrm{k}} \mathrm{C}_{\mathrm{n}, \mathrm{k} \prime}^{\mathrm{l}, \mathrm{j}}
$$

Note that the lower level implies a wider support of the basis and a fewer number of elements in the expansion of the unknown. This process can be repeated, as

$$
\begin{aligned}
& \mathrm{A}_{\mathrm{k}, \mathrm{k}^{\prime}}^{\mathrm{j}-2, \mathrm{j}}=\sum_{n} \mathrm{~g}_{\mathrm{n}-2 \mathrm{k}} \mathrm{C}_{\mathrm{n}, \mathrm{k}{ }^{\prime}}^{\mathrm{j}-1, \mathrm{j}} \\
& \mathrm{C}_{\mathrm{k}, \mathrm{k}^{\prime}}^{\mathrm{j}-2, \mathrm{j}}=\sum_{n} \mathrm{~h}_{\mathrm{n}-2 \mathrm{k}} \mathrm{C}_{\mathrm{n}, \mathrm{k}^{\prime}}^{\mathrm{j}-1, \mathrm{j}}
\end{aligned}
$$

In the same manner, the upper-right quarter

$$
\begin{aligned}
& \mathrm{B}^{\mathrm{j}, 1}=\left[\mathrm{A}^{\mathrm{j}, 1-1} \quad \mathrm{~B}^{\mathrm{j}, \mathrm{l}-1}\right], l=j, j-1, \ldots, 1 . \\
& A_{\mathrm{k}, \mathrm{k}^{\prime}}^{\mathrm{j}, \mathrm{l}-1}=\left\langle\psi_{\mathrm{l}, \mathrm{k}}, \mathrm{Z}\left(\psi_{\mathrm{j}-\mathrm{l}, \mathrm{k}^{\prime}}\right)\right\rangle \\
& \mathrm{A}_{\mathrm{k}, \mathrm{k}^{\prime}}^{\mathrm{j}, \mathrm{l}-1}=\sum_{n} \mathrm{~g}_{\mathrm{n}-2 \mathrm{k} \mathrm{k}^{\prime}} \mathrm{B}_{\mathrm{n}, \mathrm{k}^{\prime}}^{\mathrm{j}, \mathrm{l}}
\end{aligned}
$$

And;

$$
\begin{gathered}
\mathrm{B}_{\mathrm{k}, \mathrm{k}^{\prime}}^{\mathrm{j}, \mathrm{l}-1}=\left\langle\psi_{\mathrm{l}, \mathrm{k}}, \mathrm{Z}\left(\varphi_{\mathrm{l}-1, \mathrm{k}^{\prime}}\right)\right\rangle \\
\mathrm{B}_{\mathrm{k}, \mathrm{k}^{\prime}}^{\mathrm{j}, \mathrm{l}-1}=\sum_{n} \mathrm{~h}_{\mathrm{n}-2 \mathrm{k} \mathrm{k}^{\prime}} \mathrm{B}_{\mathrm{k}, \mathrm{n}}^{\mathrm{j}, \mathrm{l}}
\end{gathered}
$$

Finally, we are ready to attack the lower-right block $\mathrm{C}^{\mathrm{j}}$.

\section{WAVELET PACKET}

This section is based on the work of Pr. Stéphane Mallat in his book "A Wavelet Tour of Signal Processing the Sparse Way "Ref [1]. Wavelet packets were introduced by Coifman, Meyer, and Wickerhauser [1] by generalizing the link between multiresolution approximations and wavelets. Orthonormal wavelet packet bases are computed with conjugate mirror filters that divide the frequency axis in separate intervals of various sizes.If the signal properties change over time, it is preferable to isolate different time intervals with translated windows. A space $V_{j}$ of $a$ multiresolution approximation is decomposed in a lowerresolution space $\mathrm{V}_{\mathrm{j}+1}$ plus a detail space $\mathrm{W}_{\mathrm{j}+1}$. This is done by dividing the orthogonal basis $\left\{\varphi_{\mathrm{j}}\left(t-2^{t} n\right\}_{n \in Z}\right.$ of $\mathrm{Vj}$ into two new orthogonal bases [1-12] 


$$
\left\{\varphi _ { j + 1 } ( t - 2 ^ { t + 1 } n \} _ { n \in Z } \text { of } \mathrm { V } _ { j + 1 } \text { and } \left\{\psi_{j+1}\left(t-2^{t+1} n\right\}_{n \in Z} \text { of } \mathrm{W}_{\mathrm{j}+1}\right.\right.
$$

The decompositions of $\varphi_{\mathrm{j}+1}$ and $\psi_{\mathrm{j}+1}$ in the basis $\left\{\varphi_{\mathrm{j}}(t-\right.$ $\left.2^{t} n\right\}_{n \in Z}$ are specified by a pair of conjugate mirror filters $\mathrm{h}[\mathrm{n}]$ and $\mathrm{g}[\mathrm{n}]=(1)^{1-\mathrm{n}} \mathrm{h}[1-\mathrm{n}]$.

Theorem of Coifman, Meyer, and Wickerhauser generalizes this result to any space $\mathrm{Uj}$ that admits an orthogonal basis of functions translated by $n 2 j$ for $n \in Z$. Let $\left\{\theta_{j+1}(t-\right.$ $\left.\left.2^{t+1} n\right)\right\}_{n \in Z}$ be an orthonormal basis of a space $\mathrm{Uj}$. Let $\mathrm{h}$ and $\mathrm{g}$ be a pair of conjugate mirror filters. Define

And

$$
\theta_{j+1}^{0}(t)=\sum_{n=-\infty}^{+\infty} h[n] \theta_{j}\left(t-2^{j} n\right)
$$

$$
\theta_{j+1}^{1}(t)=\sum_{n=-\infty}^{+\infty} g[n] \theta_{j}\left(t-2^{j} n\right)
$$

The family

$$
\left\{\theta_{j+1}^{0}\left(t-2^{j+1} n\right) ; \theta_{j+1}^{1}\left(t-2^{j+1} n\right)\right\}_{n \in Z}
$$

Is an orthonormal basis of $\mathrm{Uj}$.

Theorem Coifman, Meyer, and Wickerhauser proves that we can set $\mathrm{Uj}=\mathrm{Wj}$ and divide these detail spaces to derive new bases. This space admits an orthogonal basis of scaling functions $\left\{\varphi_{\mathrm{L}}\left(t-2^{L} n\right)\right\}_{n \in Z}$ with $\varphi_{\mathrm{L}}(t)=2^{-L / 2} \varphi\left(2^{-L} t\right)$ [1].

The two wavelet packet orthogonal bases at the children nodes are defined by the splitting relations (43):

$$
\psi_{j+1}^{\mathrm{p}}(t)=\sum_{n=-\infty}^{+\infty} h[n] \psi_{j}^{\mathrm{p}}\left(t-2^{\mathrm{j}} n\right)
$$

And

$$
\psi_{j+1}^{2 \mathrm{p}+1}(t)=\sum_{n=-\infty}^{+\infty} g[n] \psi_{j}^{\mathrm{p}}\left(t-2^{\mathrm{j}} n\right)
$$

Since $\left\{\psi_{j}^{\mathrm{p}}\left(t-2^{\mathrm{j}} n\right)\right\}_{n \in Z}$ is orthonormal.

Where,

$$
h[n]=\left\langle\psi_{j+1}^{2 \mathrm{p}}(u), \psi_{j}^{\mathrm{p}}\left(u-2^{\mathrm{j}} n\right)\right\rangle
$$

and

$$
g[n]=\left\langle\psi_{j+1}^{2 \mathrm{p}+1}(u), \psi_{j}^{\mathrm{p}}\left(u-2^{\mathrm{j}} n\right)\right\rangle
$$

Any node of the binary tree is labeled by $(j, p)$, where $\mathrm{j}-\mathrm{L}$ $>=0$ is the depth of the node in the tree, and $p$ is the number of nodes that are on its left at the same depth $\mathrm{j}-\mathrm{L}$. Such a tree is illustrated in Figure 3. To each node $(j, p)$ we associate a space $\mathrm{W}_{\mathrm{j}}^{\mathrm{p}}$, which admits an orthonormal basis $\left\{\psi_{j}^{\mathrm{p}}\left(\mathrm{t}-2^{\mathrm{j}} \mathrm{n}\right)\right\}_{\mathrm{n} \in \mathrm{Z}}$ by going down the tree [1].

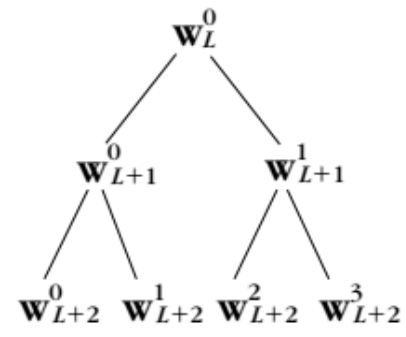

FIGURE 3. Binary tree of wavelet packet spaces[1].

\section{NUMERICAL RESULTS}

The above theory was implemented has been coded in Matlab language for calculating the induced current, and scattering pattern of the an Arbitrary Array of Parallel Wires and comparison of three methods, MoM, MoM/WT, and MoM/WPT, in terms of Sparsity of IM and CPU Time to reverse IM. The geometrical and physical parameters of a circular array of Parallel Wires are summarized in table 1. The wavelets employed is constructed from Daubechies orthogonal wavelet with vanishing moment $\mathrm{N}=4$ and Biorthogonal wavelets with vanishing moment $\mathrm{p}=2$ and $\check{p}$.

TABLE 1. Geometrical and physical parameters of an Arbitrary Array of Parallel Wires [2].

\begin{tabular}{c|c|c|c|c|c}
\hline Parameters & $\begin{array}{c}\text { No. of } \\
\text { wire }\end{array}$ & $\begin{array}{c}\text { Wire } \\
\text { radius }\end{array}$ & $\begin{array}{c}\text { Cylinder } \\
\text { radius }\end{array}$ & $\begin{array}{c}\text { wave } \\
\text { length }\end{array}$ & E0 \\
\hline Values & $\mathrm{N}=30$ & $\mathrm{Ka}=0.05$ & $\mathrm{R}=1.12 \lambda$ & $\lambda=1$ & 1 \\
\hline
\end{tabular}

The plots of Scattering pattern $\mathrm{E}(\varphi)$ against $\varphi$ for array of parallel wire are portrayed in Figs. 4.a and 4.b for the Daubechies and Biorthogonal wavelets, respectively. The results, scattering pattern, using MoM, MoM/WT, and MoM/WPT are showing and compared with published results Ref. [2-3]. We see a good agreement between three methods.

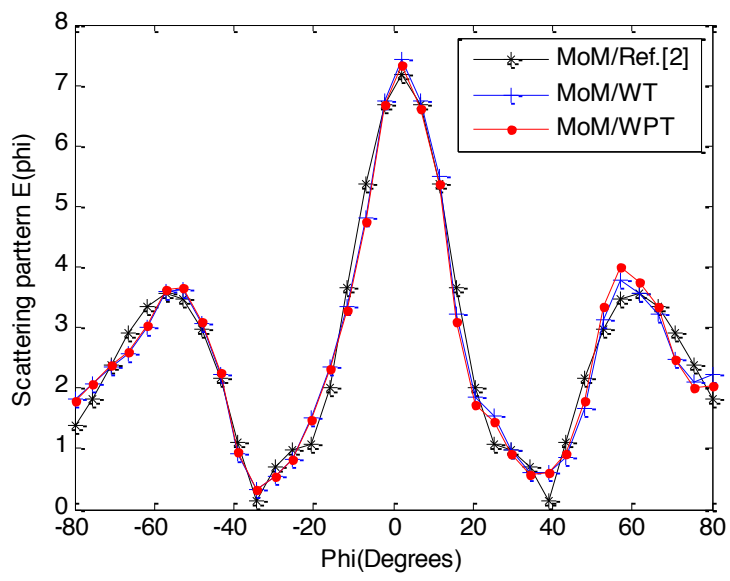

(a) 


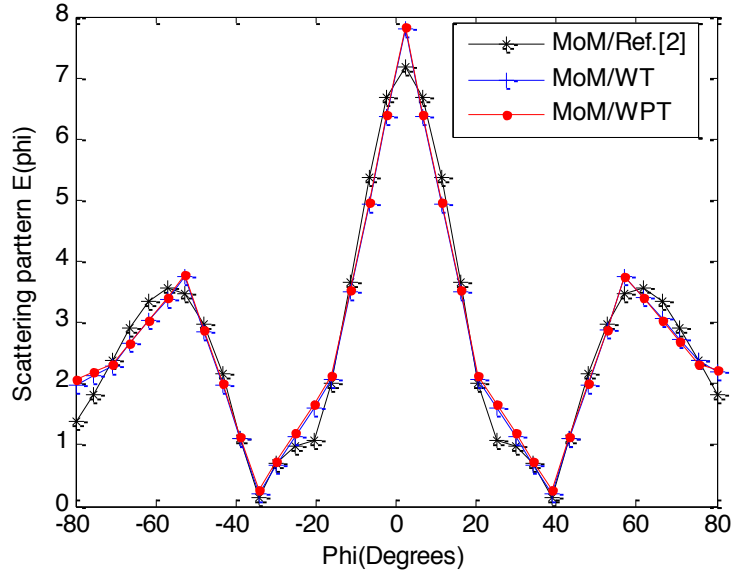

(b)

FIGURE 4. : Scattering pattern for the plane array wires with $\varphi_{0}=0$ and $\theta_{0}=\frac{\pi}{2}$ : (a) Daubechies wavelet and (b) Biorthogonal wavelet.

The comparison between the MoM/WT and MoM/WPT from the point of sparsity level as a ratio which is the percentage of the nonzero elements in the impedance matrix is presented in figures 5.a and 5.b using Daubechies and Biorthogonal wavelets, respectively.

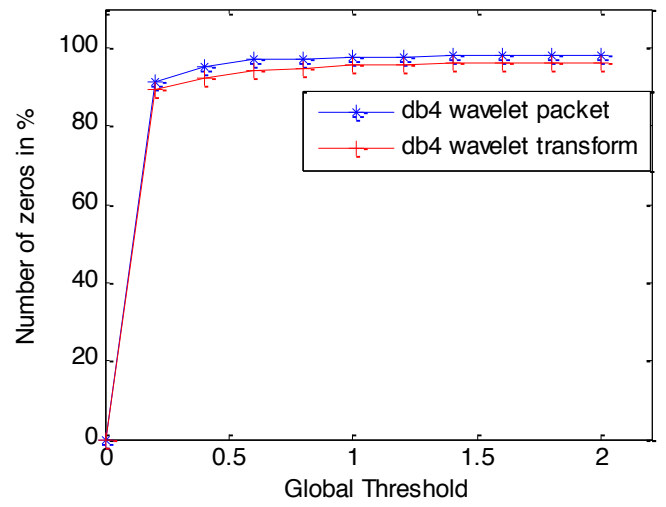

(a)

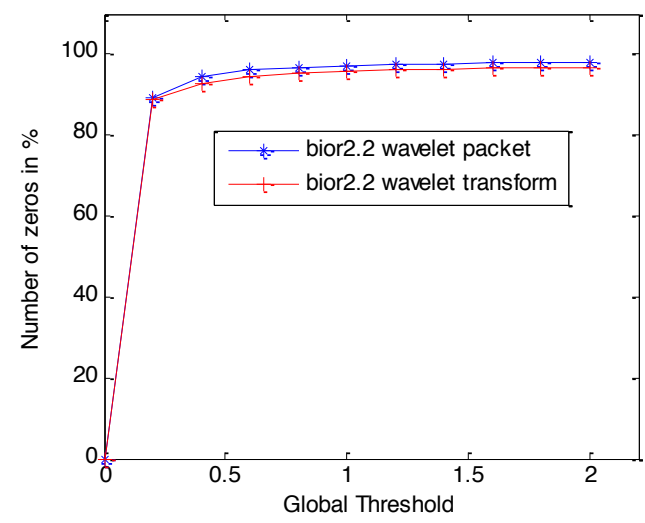

(b)

FIGURE 5. : WT and WPT matrix sparsity as a function of Threshold for the array parallels wires: (a) Daubechies wavelet and (b) Biorthogonal wavelet.
As seen in Figures 5, the MoM/WPT method gives the matrix sparsity more than the MoM/WT method, for example for $\mathrm{Thr}=0.6$ the MoM / WPT the rate of sparsity is $97 \%$ while for $\mathrm{MoM} / \mathrm{WT}$ is $94 \%$ for the Daubechie wavelet, the same for the Biorthogonal wavelet. We observe also that the number of zeros in IM increases when increasing the threshold; this is due to canceling of the impedance matrix elements.

In order to better explain the benefits of the performed computational model, the following quantities have been also considered [19]:

$$
\operatorname{err}_{r}(t h r)=\frac{\left\|Z_{W}-Z_{m o m}\right\|_{2}}{\left\|Z_{m o m}\right\|_{2}} \text { and } \quad m e m=\frac{N_{W}}{N m o m}
$$

Where Zmom, Nmom are the computed solution and number of matrix entries with $\mathrm{MOM}$ and $\mathrm{Zw}, \mathrm{N}_{\mathrm{W}}$ the solution impedance and number of matrix entries with wavelet-MoM and the symbol \|\|$_{2}$ denotes the $\mathrm{L}^{2}$ norm.

Table 2 shows a comparison CPU Time, CPU Time reduction, relative error and memory requirement using $\mathrm{MoM} / \mathrm{WT}$ and MoM/WPT methods associated with Daubechies and Biorthogonal wavelets in characterizing array parallel wires. The calculations are made on a PC computer with AMD Dual-Core $1.30 \mathrm{GHz}$ CPU, 2 GB memory and Windows 7 Professional system.

TABLE 2. Comparison CPU Time, CPU Time reduction, relative error and memory requirement.

\begin{tabular}{cccc}
\hline Parameters & Wavelets & Daubechies & Biorthogonal \\
\hline CPU (ms) & MoM/WT & 17.44 & 17.79 \\
& MoM/WPT & 17.16 & 16.98 \\
CPU Time & MoM/WT & 1.84 & 2.19 \\
$\begin{array}{c}\text { Io reverse } \\
\text { CPU }\end{array}$ & MoM/WPT & 1.56 & 1.38 \\
reduction \% & MoM/WT & 12.80 & 11.05 \\
& MoM/WPT & 14.2 & 15.1 \\
mem \% & MoM/WPT & 69.86 & 70.26 \\
Relative & MoM/WT & 0.0920 & 0.0913 \\
error & MoM/WPT & 0.0887 & 0.0876 \\
& & &
\end{tabular}

Table 2 also reveals that the use of MoM/WT and $\mathrm{MoM} / \mathrm{WPT}$ leads to a CPU Time reduction of $11.05 \%$ and $15.10 \%$, respectively, for a threshold of 0.6. This is due to the fact that the CPU time to invert a matrix is proportional to the number of non-zero matrix elements. On the other hand, we can notice that the MoM/WPT(db4) given relative error less than MoM/WT.

\section{CONCLUSIONS}

In this paper, the scattering from a circular array of parallel wires has been analyzed using a numerical technique based 
on the MoM and wavelets. In the first, the wavelet transforms and wavelet packet transforms have been applied to the MoM matrix in order to obtain a sparseness in the system matrix. In the second, a comparison among these techniques, MoM/WT and MoM/WPT, is presented using numerical examples. Several plots for the Scattering pattern presented, regarding various methods. The computed results were compared with published results Ref. [2-3] and very good agreement was observed. In summary, the above-described use of wavelet packet transformations for the solution of electromagnetic integral equations appears to be superior in all aspects of comparison including characterization of the matrix sparsity, and computation time.

\section{REFERENCES:}

[1] Stéphane Mallat, "A Wavelet Tour of Signal Processing the Sparse Way", Third Edition. Copyright (2009) by Elsevier Inc

[2] Matthew N. O. Sadiku, Ph.D., "Numerical techniques in electromagnetics", 2nd Ed, (C2001 by CRC Press LLC, Boca Raton London New York Washington, D.C.

[3] J.H. Richmond, "Scattering by an arbitrary array of parallel wires," IEEE Trans. Micro. Theo. Tech., vol. MTT-13, no. 4, July 1965, pp. 408-412.

[4] Walton C. Gibson, "The Method of Moments in Electromagnetics", Second Edition, (C) (2015) by Taylor \& Francis Group, LLC.

[5] R. F. Harrington, Field computation by Moment Methods, Editorial Board William Perkins, Editor in Chief 1992.

[6] N. Khanna, V. Kumar and S. K. Kaushik, "Wavelet packets and their vanishing moments", Poincare Journal of Analysis \& Applications, Vol. 2017(2), 95-105.

[7] J. C. Goswami, A. K. Chan, "Fundamentals Wavelets Theory, Algorithms, and Applications", Copyright (C) (2011) by John Wiley \& Sons, Inc. All rights reserved.

[8] G. W. Pan, "Wavelets in Electromagnetics and Device Modeling", Copyright (C) (2003) by John Wiley \& Sons, Inc. All rights reserved.

[9] Mohammad Yazdi And Nader Komjani, "Polarizability calculation of arbitrary individual scatterers, scatterers in arrays, and substrated scatterers", Journal of the Optical Society of America B, Vol. 33, No. 3 / March 2016.

[10] Mohamed Bayjja and al, "Modeling a Planar Coupled Microstrip Lines using various Wavelets and Method of Moments", Advanced Electromagnetics, Vol. 9, No. 1, March 2019.

[11] P. Papakanellos, "Study of Two Arbitrarily Located Parallel Cylindrical Dipoles Based on an Auxiliary Sources Technique", Electromagnetics, 2003, 23:5, 399-416. DOI:10.1080/02726340390203153.

[12] Wojciech L. Golik, "Wavelet Packets for Fast Solution of Electromagnetic Integral Equations", IEEE Trans. on Antennas and Propagat., Vol. 46, No. 5, May 1998.

[13] Dorsaf Omri, Mourad Aidi - Taoufk Aguili, "A comparison of three temporal basis functions for the time-domain method of moments (TD-MoM)", Journal of Computational Electronics, March 2020. https://doi.org/10.1007/s10825-020-01468-8.

[14] mir Geranmayeh, Rouzbeh Moini, and S. H. Hesam Sadeghi, Numerical Simulation of Electromagnetic Fields Radiated by Lightning Return Stroke Channels: A Wavelet-Based Approach, IEEE Trans. on Electromag. Compatibility., Vol. 48, No. 1, Feb. 2006

[15] E. Ashpazzadeh, B. Han, M. Lakestani, Biorthogonal multiwavelets on the interval for numerical solutions of Burgers' equation, Journal of Computational and Applied Mathematics, 2016.

http://dx.doi.org/10.1016/j.cam.2016.11.045.

[16] Mohamed Bayjja, A.K. Belbachir, M. Boussouis, and Naima Amar Touhami, "Orthogonal and biorthogonal compactly supported wavelets in modeling the circular loop antenna", International Journal of Microwave and Optical Technology,VOL. 12, NO. 5, September 2017.

[17] D.B. Davidson, "Computational Electromagnetics for $\mathrm{Rf}$ and Microwave Engineering", Published in the United States of America by Cambridge University Press, New York, 2005.

[18] M. Lerer, A.B. Kleshchenkov, O.S. Labunko, "Time-Domain Scattering from Arbitrary Array of Parallel Electric Dipoles", 11 Int Conf. on Mathematical Methods in Electromagnetic Theory. Kharkiv, Ukraine, June 26-29, 2006.

[19] Guido Ala, and al, "An Advanced Numerical Model in Solving Thin-Wire Integral Equations by Using Semi-Orthogonal Compactly Supported Spline Wavelets", IEEE Trans. on Electromag. Compatibility, Vol. 45, No. 2, May 2003. 